ORAL PATHOLOGY

\section{A 4-year prospective study on epidemiology and clinicopathological presentation of odontogenic tumours in Tanzania}

Simon ENM, Merkx MAW et al. Oral Surg 2003; 99: 598-602

In this black African population, the incidence of ameloblastoma did not differ from that of European populations.

A number of retrospective studies have suggested that black Africans experience ameloblastoma more frequently than other ethnic groups. Over a 4 year period, 116 patients were seen with odontogenic tumours in a Dar es Salaam clinic, as a result of a request for referral of all such patients seen in dental departments in Tanzania. Diagnoses were confirmed by two pathologists, and following treatment, 51 ameloblastoma patients were followed up from 6 months to 4 yrs. In that time, 2 patients had recurrences.

The majority of all tumours were ameloblastomas (80\%), followed by odontogenic myxoma (7\%), odontoma (3\%), and 1-2\% each of 7 other tumours. About $2 / 3$ presented in the 2 nd and $3 \mathrm{rd}$ decades. Pain was present in $20 \%$ of ameloblastoma patients, and inferior alveolar paraesthesia in 8\%. Half of ameloblastomas were follicular (52\%) and 24\% were plexiform. Two ameloblastic carcinomas were diagnosed, but without apparent spread to nodes or metastases. Incidence of ameloblastoma was calculated as 0.68 per million per year in the 34 million population of Tanzania. This is similar to its incidence in European populations.

doi:10.1038/sj.bdj.4812698

\section{IMPLANT DENTISTRY; PROSTHODONTICS}

A prospective 3-year study of fixed bridges linking Astra Tech ST implants to natural teeth Palmer RM, Howe LC et al. Clin Oral Imp/ Res 2005; 16: 302-307

Bridges supported on both teeth and implants were stable at 3 yrs.

Implants are held rigidly in bone, whereas teeth are able to move within the allowance of the periodontal ligament. Because of unsatisfactory results from both retrospective and prospective studies, some authorities suggest that bridges supported on both teeth and implants are likely to have complications.

In this prospective study, 6 patients were recruited with a free end saddle in the maxilla, and 15 with one in the mandible. Two subjects dropped out of the study. All had opposing natural teeth or tooth-supported bridges, and were non-smokers. Implants were placed in a 2 stage procedure, and 3-unit bridges were cemented, 6 with distal cantilever pontics, and 13 with fixed-fixed design. At 3 years, all abutments had stable bone levels, with no intrusion of teeth. In 8 cases, temporary cement allowed de-bonding, and permanent re-cementation was required. Other minor complications occurred. The authors suggest that this type of treatment is useful in posterior areas of limited bone volume.
GERODONTICS; SPECIAL CARE DENTISTRY

\section{Effectiveness of health care worker training on the oral health of elderly residents of nursing homes}

Nicol R, Sweeney MP et al. Community Dent Oral Epidemio/ 2005; 33: 115-124

A staff training programme led to improved oral health of residents.

Provision of dental care and oral hygiene support is frequently considered inadequate for elderly institutionalised patients. In 3 nursing homes and 2 long-stay hospitals in Caithness, 78 patients (median age $84 \mathrm{yrs}$ ) were selected for a trial of a carer educational programme, 39 in the experimental group (E) and 39 in the control (C). After selection, oral health was assessed in subjects, and subsequently training was given to staff providing care for the E group.

At initial assessment, 58\% were non-smokers, and 26\%, exsmokers. Groups had similar numbers of teeth and complete or partial dentures, and about half the subjects in each group took medications which might cause xerostomia. Reassessment occurred at 3, 9 and 18 months, and $\mathrm{C}$ group staff were also trained after the 9 month assessment. At 3 months, significant improvements were found in the E group in oral hygiene measures, mucosal disease, angular cheilitis, continuous denture-wearing and denture stomatitis. By the final assessment, 8 subjects had died in each group. In $\mathrm{C}$ group, oral hygiene measures and continuous denture-wearing had improved at 18 months.

doi:10.1038/sj.bdj.4812700

ORAL PATHOLOGY; PROSTHODONTICS

Risk factors associated with denture stomatitis in the United States

Shulman JD, Rivera-Hidalgo F et al. J Oral Pathol Med 2005; 34: 340-346

Denture stomatitis (DS) was more frequent in patients wearing dentures continuously, in those with low levels of vitamin A, and in smokers.

This study examined DS data from the US NHANES III study, using a multi-stage sample of 3,450 patients wearing at least 1 removable denture. DS lesions were present in $28 \%$ of subjects. Multivariate analysis was used to explore possible associations with risk factors, for maxillary and mandibular complete (MXC and MDC) and partial (MXP and MDP) dentures.

The final model identified significant adjusted odds ratios of 6.2 (MXC) and 5.2 (MDC) for wearing dentures continuously, of 5.8 (MDC), 5.7 (MXP) and 24.4 (MDP) for vitamin A deficiency, of 0.4 (MXC) and 0.5 (MDC) for inadequate reline material, of 2.3 (MDC) for poor retention, of 2.1 (MXP) for instability, of 1.3 (MXC), 1.5 (MDC) and 2.0 (MXP) for $>15$ cigarettes/day, and 2.6 (MXP) for age below 40 yrs. The authors recommend improved denture cleaning and removing dentures at night, and state that their vitamin A findings need to be confirmed in other studies. 